\title{
Suppression of photoconductivity by magnetic field in epitaxial manganite thin films
}

\author{
E. J. Guo, ${ }^{1}$ L. Wang, ${ }^{1}$ H. B. Lu,,${ }^{1}$ a) H. Z. Guo, ${ }^{1}$ M. He, ${ }^{1}$ K. J. Jin,,${ }^{1}$ G. Z. Yang, ${ }^{1}$ \\ and J. Gao ${ }^{2}$ \\ ${ }^{1}$ Beijing National Laboratory for Condensed Matter Physics, Institute of Physics, \\ Chinese Academy of Sciences, Beijing 100190, China \\ ${ }^{2}$ Department of Physics, The University of Hong Kong, Pokfulam Road, Hong Kong
}

(Received 11 December 2011; accepted 23 January 2012; published online 6 February 2012)

\begin{abstract}
The erasure of photoinduced resistance (PR) by the magnetic field was investigated in manganite films. The PR was significantly suppressed when a magnetic field was introduced at low temperature. The decrease (or increase) of PR with increment of magnetic field was observed in ferromagnetic (or paramagnetic) phases of films, respectively. Our results are suggested to be the coaction of two effects under magnetic fields: (i) the reorientation of domains and spin directions of photoexcited carriers and (ii) electrons trapped around oxygen vacancies released and recombined with majority carriers in films. The interplay of the external fields is a good demonstration of the strong coupling between spins and charges in colossal magnetoresistance materials. (C) 2012 American Institute of Physics. [doi:10.1063/1.3683551]
\end{abstract}

The doped manganite oxides has been intensively studied due to its rich physical properties and the potential in device applications. The features of the magnetic and transport properties of this system can be well understood in the framework of the double-exchange (DE) mechanism and electron-phonon interaction due to the Jahn-Teller (J-T) distortion. $^{1,2}$ One of the interesting properties in manganites is that they are sensitive to the external fields and factors, such as magnetic field, ${ }^{3}$ electric field, ${ }^{4}$ strain, ${ }^{5,6}$ and light. ${ }^{7-13}$ In the past decades, photoinduced effects, such as photoinduced insulator-to-metal (IM) transition, ${ }^{7}$ transient and persistent photoconductivity, ${ }^{8}$ and photoinduced demagnetization, ${ }^{9-13}$ had been widely studied. Among the photoinduced effects, the photoinduced demagnetization effect in the doped manganites is a fascinating phenomenon. It is believed that the photoexcited $e_{\mathrm{g}}$ carriers are expected to significantly affect the magnetic interaction via modifying the $t_{2 \mathrm{~g}}$ spin ordering. Meanwhile, the transport property of manganites is strongly influenced by the photoinduced demagnetization effect at low temperature. In $\mathrm{La}_{0.7} \mathrm{Ca}_{0.3} \mathrm{MnO}_{3}$ films, the increase of resistance under laser illumination was observed in ferromagnetic phase, indicating that the photoexcited carriers changed the antiferromagnetic/ferromagnetic phase balance, favoring the insulating antiferromagnetic phase..$^{9,12,13}$ In previous works, we reported the strain-mediated photoinduced demagnetization effect in manganites via external applied electric field on the ferroelectric substrates. ${ }^{6}$ However, the related physical mechanism is not very clear so far. In this letter, we studied the photoinduced change of the resistance (PR) of $\mathrm{La}_{0.8} \mathrm{Ca}_{0.2} \mathrm{MnO}_{3}$ (LCMO) thin films under the external magnetic field. The results show that the proportion of PR is weakened by the external magnetic field. The mechanism of such behavior was discussed in detail. The results provide us some insight into the nature of physical properties of manganites under irradiation of light.

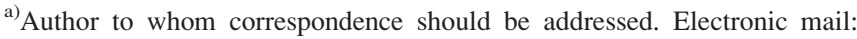
hblu@aphy.iphy.ac.cn.
}

The LCMO thin films with thickness of $\sim 150 \mathrm{~nm}$ were epitaxially grown on the $\mathrm{SrTiO}_{3}$ (STO) (001) substrates by pulsed laser deposition (PLD) using a $\mathrm{KrF}$ excimer laser with a wavelength of $248 \mathrm{~nm} .^{6}$ The laser pulse frequency was $2 \mathrm{~Hz}$ and the energy density of laser pulse was $\sim 2 \mathrm{~J} / \mathrm{cm}^{2}$. The temperature of substrate was kept at $700^{\circ} \mathrm{C}$, and the oxygen pressure was maintained at 0.3 mbar during the deposition. After the deposition, the LCMO films were in situ annealed in oxygen atmosphere with $1 \mathrm{~atm}$ pressure for $20 \mathrm{~min}$. The crystallization of the LCMO films was examined by $\mathrm{x}$-ray diffraction (XRD). The XRD results demonstrated that the films are in single phase with $c$-axis orientation. The sample was cut into $5 \times 5 \mathrm{~mm}^{2}$ and $1.5 \times 1.5 \mathrm{~mm}^{2}$ for the electrical and magnetic measurements, respectively. For electrical measurement, in order to obtain the Ohmic contact, four silver contact pads were prepared on LCMO films by thermal evaporation and the current leads were connected to the silver pads using supersonic wire bonder. The resistance of LCMO films was measured by standard four-probe method using a physical property measurement system (PPMS) with an optical window. The inset of Fig. 2(a) shows the schematic diagram of the measuring configuration with respect to the illuminated region. The direction of magnetic field was applied perpendicularly to the film surface (ab-plane). The dc magnetization measurements were carried out on a magnetic property measurement system (MPMS) using a fiberoptic sample holder (FOSH) with the diameter of $1.6 \mathrm{~mm}$, which allowed light can incident to the films directly through a fiberoptic rod and bundle during the magnetic measurements. The light source used in the experiment was a He-Ne laser with the wavelength of $632.8 \mathrm{~nm}$ and the maximum power density of $\sim 5 \mathrm{~mW} / \mathrm{cm}^{2}$.

Figure 1(a) shows the temperature dependence of resistances for LCMO films under various magnetic fields of 0,1 , $3,5,7$, and $9 \mathrm{~T}$ measured in dark, respectively. At zero magnetic field, the resistances increase as the temperature decrease and undergo a sharp drop at $T_{\mathrm{C}} \sim 190 \mathrm{~K}$, demonstrating an IM transition. As further increasing the magnetic 


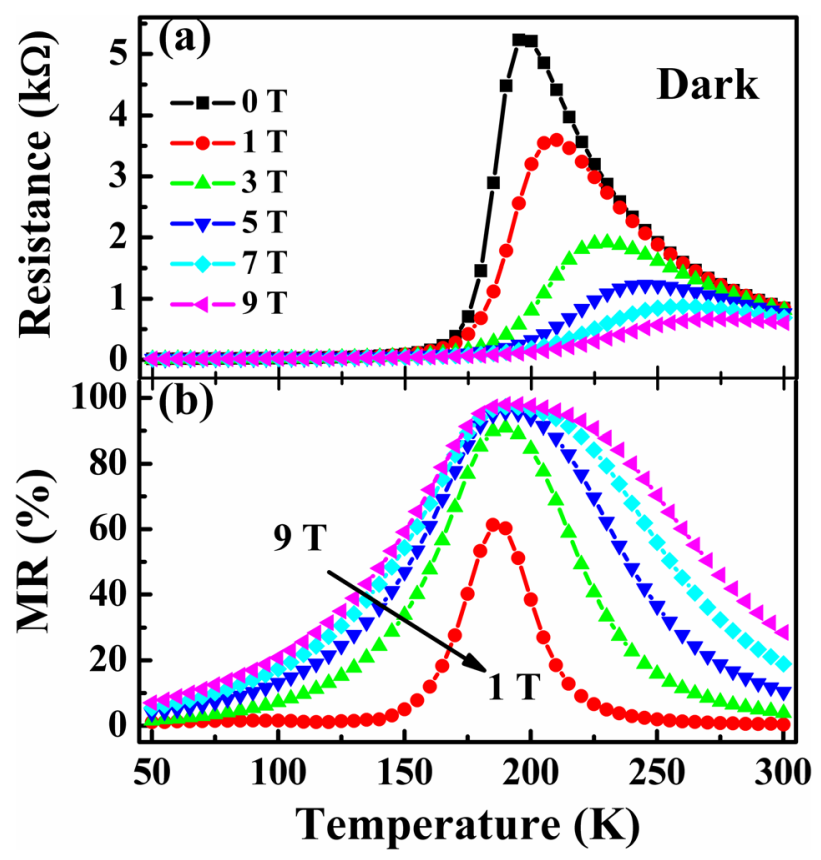

FIG. 1. (Color online) The temperature dependence of (a) resistance and (b) magnetoresistance $(\mathrm{MR})$ (defined as $\mathrm{MR}=[\mathrm{R}(0)-\mathrm{R}(\mathrm{H})] / \mathrm{R}(0) \times 100 \%$ ) for LCMO film under various fields of $0,1,3,5,7$, and $9 \mathrm{~T}$ when measured in dark.

field, the resistances decrease greatly in a broad range of temperature and the $T_{\mathrm{C}}$ shifts to higher temperature. These are the typical colossal magnetoresistance (MR) characteristics of the doped manganites. The temperature dependence of $\mathrm{MR}$ (defined as $\mathrm{MR}=[\mathrm{R}(0)-\mathrm{R}(\mathrm{H})] / \mathrm{R}(0) \times 100 \%$ ) under various magnetic fields is presented in Fig. 1(b). The peak of MR is clearly correlated with the IM transition temperature. The maximum MR are $60.2 \%, 90.9 \%, 95.7 \%, 97.3 \%$, and $98 \%$ under the magnetic fields of $1,3,5,7$, and $9 \mathrm{~T}$ at $190 \mathrm{~K}$, respectively.

The temperature dependence of resistances for LCMO films is also measured under the irradiation of light without magnetic field. Figure 2(a) shows the temperature dependence of $\mathrm{PR}$ (defined as $\left.\mathrm{PR}=\left(\mathrm{R}_{\text {light }}-\mathrm{R}_{\text {dark }}\right) / \mathrm{R}_{\text {dark }} \times 100 \%\right)$ under the irradiation of light with different power density. The PR is negative when LCMO is in paramagnetic insulating (PMI) phase, and the PR turns to positive when LCMO is in ferromagnetic metallic (FMM) phase. As shown in Fig. 2(a), PR decreases from $6.1 \%, 4.6 \%$, and $2.1 \%$ to $0.9 \%$ at $180 \mathrm{~K}$, while PR increases from $-0.76 \%,-0.53 \%$, and $-0.21 \%$, to $-0.08 \%$ at $215 \mathrm{~K}$ when the laser power density decreases from $5,2.5$, and 0.5 to $0.05 \mathrm{~mW} / \mathrm{cm}^{2}$, respectively. The positions of the minimum and maximum PR are almost fixed for the different laser power density. The phenomena can be understood as following: the transport of doped manganites is closely related to the spin of $e_{\mathrm{g}}$ carriers and localized $t_{2 \mathrm{~g}}$ spin core in $\mathrm{Mn}^{3+}$ and $\mathrm{Mn}^{4+}$ ions. In the PMI phase, the transport of LCMO can be described by small polaron hopping conduction model. The injection of photoexcited carriers can enhance the hopping of small polarons, leading to a decrease of resistance. In the FMM phase, light can stimulate electrons from localized $t_{2 \mathrm{~g}}$ state to travelling $e_{\mathrm{g}}$ state. As a result, the DE effect will be weakened, and the resistance of LCMO films will be increased. Figure 2(b)

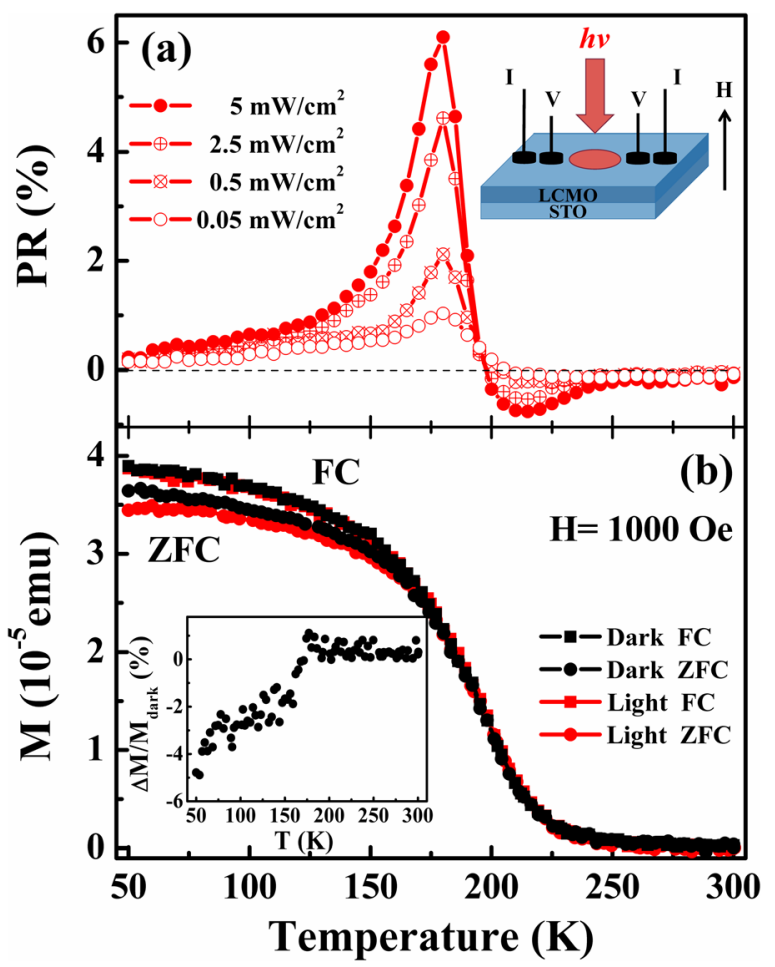

FIG. 2. (Color online) (a) The temperature dependence of the photoinduced change of the resistance $(\mathrm{PR})$ (defined as $\mathrm{PR}=\left(\mathrm{R}_{\text {light }}-\mathrm{R}_{\text {dark }}\right) / \mathrm{R}_{\text {dark }} \times 100 \%$ ) for LCMO film when measured under the irradiation of He-Ne laser with different power density. The inset shows the schematic diagram of the measuring configuration with respect to the illuminated region. The direction of magnetic fields was applied perpendicularly to the film surface. (b) The temperature dependence of the ZFC and FC magnetizations in LCMO film under a field of $1000 \mathrm{Oe}$ when measured in dark and irradiation of $\mathrm{He}-\mathrm{Ne}$ laser, respectively. The inset shows the temperature dependence of magnetization differences $\Delta \mathrm{M}(\mathrm{T}) / \mathrm{M}^{\text {dark }}{ }_{\mathrm{ZFC}}(\mathrm{T})$, where we defined $\left.\Delta \mathrm{M}(\mathrm{T})=\mathrm{M}^{\text {light }}{ }_{\mathrm{ZFC}}(\mathrm{T})-\mathrm{M}_{\mathrm{ZFC}}^{\text {dark }}(\mathrm{T})\right)$.

presents the temperature dependence of the magnetization with and without irradiation of light under a field of 1000 Oe measured in field-cooling (FC) and zero-field-cooling (ZFC) modes. The PM to FM transition temperature is at $\sim 195 \mathrm{~K}$, corresponding to the IM transition temperature. From the ZFC curves, one can see that the magnetic moment in ZFC with irradiation of light $\mathrm{M}^{\text {light }}{ }_{\mathrm{ZFC}}(\mathrm{T})$ is smaller than that of in $\mathrm{ZFC}$ in dark $\mathrm{M}_{\mathrm{ZFC}}^{\text {dark }}(\mathrm{T})$ in FM phase. The temperature dependence of the photoinduced magnetization difference $\Delta \mathrm{M}(\mathrm{T})$ (defined as $\Delta \mathrm{M}(\mathrm{T})=\mathrm{M}^{\text {light }}{ }_{\mathrm{ZFC}}(\mathrm{T})-\mathrm{M}^{\text {dark }}{ }_{\mathrm{ZFC}}(\mathrm{T})$ ) is shown in the inset of Fig. 2(b). The $\Delta \mathrm{M}(\mathrm{T}) / \mathrm{M}_{\text {dark }}(\mathrm{T})$ decrease by $\sim 5 \%$ at $50 \mathrm{~K}$. The small difference in $\Delta \mathrm{M}(\mathrm{T})$ observed in the experiment may due to the weak light density of $\sim 5 \mathrm{~mW} / \mathrm{cm}^{2}$. The decreasing of magnetization in FM phase indicates that the magnetic phase has been modified by the illumination due to the photoinduced demagnetization effect. The magnetic measurements give direct evidence that the photoexcited $e_{\mathrm{g}}$ carriers suppress the spin correlation resulting in the decrease of the macroscopic magnetization.

To test whether the photoinduced demagnetization effect in LCMO films can be influenced by magnetic fields, we further measured the resistance of LCMO under the magnetic field from 1 to $9 \mathrm{~T}$ with the irradiation of light. The difference of resistances measured in dark and the light illumination (referred to $R_{\text {light }}-R_{\text {dark }}$ ) under different magnetic fields is shown in the Fig. 3(a). The absolute value of 

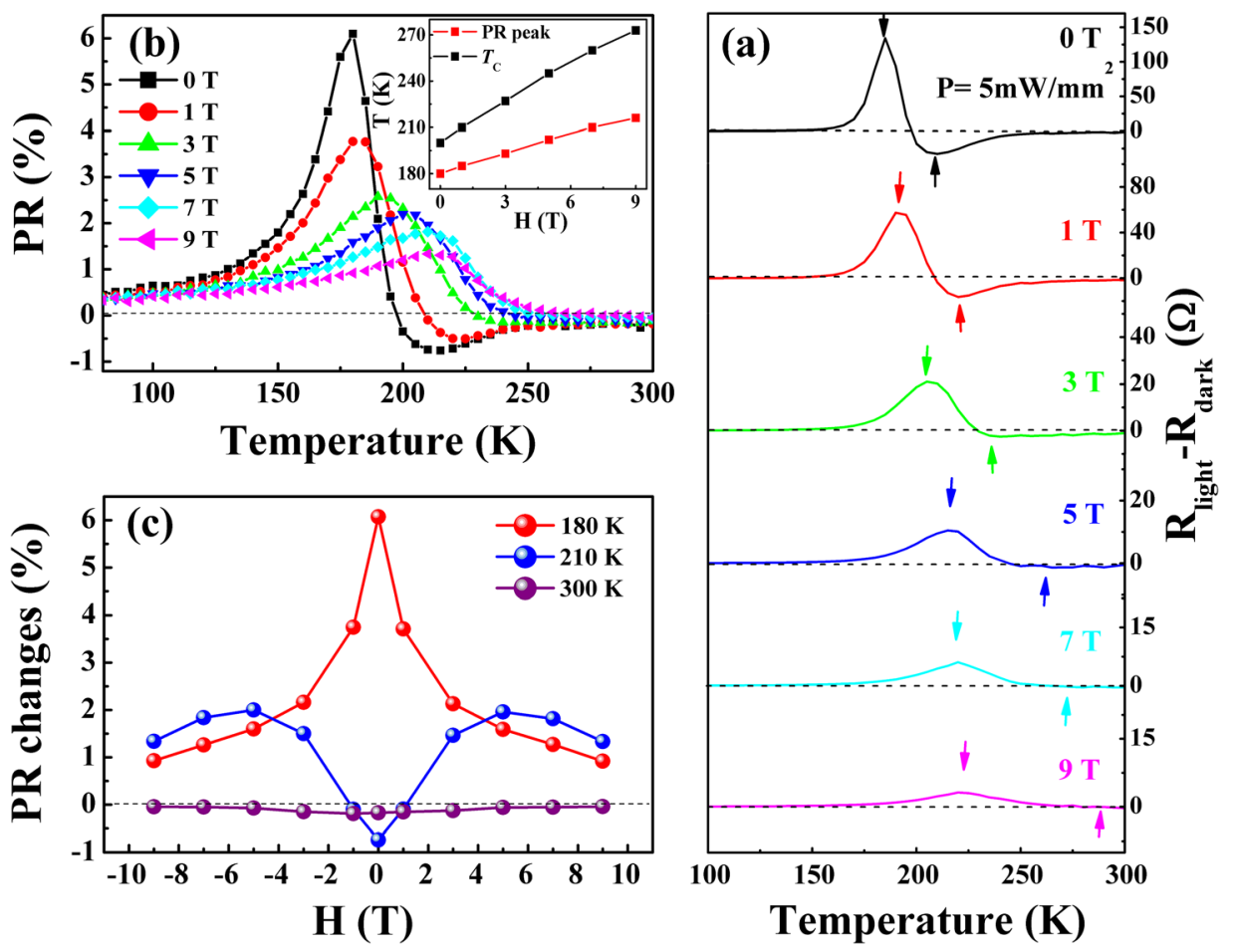

FIG. 3. (Color online) The temperature dependence of (a) $R_{\text {light }}-R_{\text {dark }}$ and (b) PR for LCMO film under various fields of $0,1,3$, 5,7 , and $9 \mathrm{~T}$, respectively. The arrows in (a) point out the positions of the maximum and minimum PR. The inset of (b) shows the field dependence of the metal-to-insulator transition temperatures $T_{\mathrm{C}}$ and the temperatures at positive PR peaks. (c) The field dependence of PR measured in 180, 210, and $300 \mathrm{~K}$, respectively.
$R_{\text {light }}-R_{\text {dark }}$, no matter negative or positive it is, decreases greatly as magnetic fields are applied. The positions of the minimum and maximum values of $R_{\text {light }}-R_{\text {dark }}$ shift to higher temperature with further increasing the magnetic fields. Figure 3(b) shows the temperature dependence of the PR under various magnetic fields. The maximum PR are $6.1 \%$ at $180 \mathrm{~K}, 3.78 \%$ at $183 \mathrm{~K}, 2.57 \%$ at $190 \mathrm{~K}, 2.2 \%$ at $200 \mathrm{~K}$, $1.81 \%$ at $210 \mathrm{~K}$, and $1.3 \%$ at $215 \mathrm{~K}$ under the magnetic fields of $0,1,3,5,7$, and $9 \mathrm{~T}$, respectively. The maximum PR was reduced about five times when a magnetic field was introduced at low temperature. The inset of Fig. 3(b) shows the linearly growth of $T_{\mathrm{C}}$ and the positive PR peaks with the increasing of temperature and the magnetic fields. As shown in the Fig. 3(c), the PR will decrease continually with the magnetic field increasing when measured at $180 \mathrm{~K}$, where is in the FM phases. However, when measured at $210 \mathrm{~K}$, the PR will increase at first from negative to positive as magnetic field increase from 0 to $5 \mathrm{~T}$, then PR decrease with the magnetic field further increasing from 5 to $9 \mathrm{~T}$. Different from the above results, the PR increase from the minimum value approaching zero with magnetic field increasing when measured at $300 \mathrm{~K}$. The results reveal that the PR can be decreased by the magnetic field and the resistance tends to its original values of no light irradiation with the increase of the magnetic field. The similar results were reported on $\mathrm{La}_{2 / 3}$ $\mathrm{Sr}_{1 / 3} \mathrm{MnO}_{3-\delta}$ by Sheng et al. ${ }^{11}$ It was found that the resistance shows a quick increase after applying a small magnetic field, while the resistance of films shows a sharp decrease contrarily when a larger magnetic field is applied.

As mentioned above, we think the different PR behavior indicates the existence of the different mechanisms affects the electric properties of LCMO, which is closely related to the magnetic property of manganite films. There are two possible factors coexisted in the light irradiated LCMO films once the magnetic field was applied. Firstly, the external magnetic fields would reorient the FMM domains and spin directions of photoexcited carriers in LCMO films. In a strong magnetic field, the magnetic domains tend to align along the external field direction, resulting in the electrons transferring more easily across the domain-wall boundaries. At the same time, some fractions of PMI phase will convert to FMM phase under magnetic field, leading to the formation of a complete conduction path. ${ }^{14,15}$ Moreover, as we all know, the efficiency of photoinduced demagnetization is strongly dependent on the spin direction of the excited $e_{\mathrm{g}}$ carriers. The original spin direction of the excited $e_{\mathrm{g}}$ carriers is aligned randomly at zero field, while it will be aligned to the same direction under the external magnetic fields. This will cause photoinduced carriers transfer freely. All the above mechanisms will contribute to the decrease of resistance in LCMO, resulting in the suppressing of PR. The second effect brought out by the magnetic field is the release and recombination of electrons trapped in the oxygen vacancies. ${ }^{11}$ External magnetic field would lead to the growth of FMM phase at the expense of PMI phase. With melting the PMI phase around the oxygen vacancies, the electrons trapped by oxygen vacancies will possibly recombine with the holes, which are the majority carriers in LCMO films. It gives rise to the decrease of carrier density leading to the increase of resistance in LCMO films. Furthermore, applied magnetic fields will strongly affect the small polaron hoping energy in the PMI phase. The difference in the mobility of the initial (polaron) state and final (band) states should reduce as FMM phase in LCMO grows when magnetic fields increase. The localized small polaron in the PMI phase gradually transforms into an itinerant large polaron in the FMM phase. Therefore, the small polaron hoping energy of LCMO will gradually reduce as magnetic fields increase, resulting in 
the reduction of resistance changes. Therefore, in the FMM phase, the former factor is dominant, resulting in the decrease of PR. However, in the PMI phase, the later factor will play a major role. Thus, the PR in LCMO film will increase with the magnetic field increasing.

To further confirm the erasure of PR by magnetic field, the similar experiment was carried out on the LCMO films grown on the $\mathrm{LaAlO}_{3}$ (LAO) substrate and the similar tendency of PR was observed. The resistance of LCMO films grown on LAO substrate is one degree of magnitude higher than that grown on STO substrate, and the IM transition temperature is about $200 \mathrm{~K}$. Figure 4 shows the temperature dependence of $R_{\text {light }}-R_{\text {dark }}$ for LCMO films grown on LAO substrate under various fields of $0,1,3,5,7$, and $9 \mathrm{~T}$, respectively. Without the magnetic field, the maximum $\mathrm{PR}$ is $1.45 \%$ at $170 \mathrm{~K}$ and the minimum PR is $-0.74 \%$ at $215 \mathrm{~K}$, respectively. We find that the PR is also suppressed as magnetic fields increasing. The inset of Fig. 4 displays that the PR changes are $1.45 \%$ and $0.045 \%$ under 0 and $9 \mathrm{~T}$ at $170 \mathrm{~K}$, respectively. The maximum PR is reduced over thirty times when magnetic fields increase from 0 to $9 \mathrm{~T}$ at $170 \mathrm{~K}$. It is note that the PR of LCMO films grown on LAO substrates is relative lower than that grown on STO substrates. Here, the strain effect induced by the different substrates should take into account. According to the XRD results, an in-plane tensile strain will be introduced in the LCMO film due to the lattice mismatch of $0.72 \%$ between STO substrate and LCMO film. While, a larger compressive in-plane strain is achieved due to the larger lattice mismatch of $-3.42 \%$ between LAO substrate and LCMO film. As discussed in our previous works, the reduction of in-plane strain of LCMO will influence the J-T distortion of $\mathrm{MnO}_{6}$ octahedral and further weaken the electron-lattice coupling. ${ }^{16}$ Therefore, a smaller strain will result in a reduction of the separation between upper $t_{2 \mathrm{~g}}$ and lower $e_{\mathrm{g}}$ levels, and the observed larger PR in LCMO films grown on STO substrate is understandable. In addition, as mentioned above, magnetic domains will be reoriented under external magnetic fields. It is well known that the domain characteristics are strongly dependent on the film thickness. ${ }^{17-19}$ Bakaul et al. ${ }^{19}$ found that thinner $\mathrm{La}_{0.7} \mathrm{Sr}_{0.3} \mathrm{MnO}_{3}$ films exhibit long and continuous

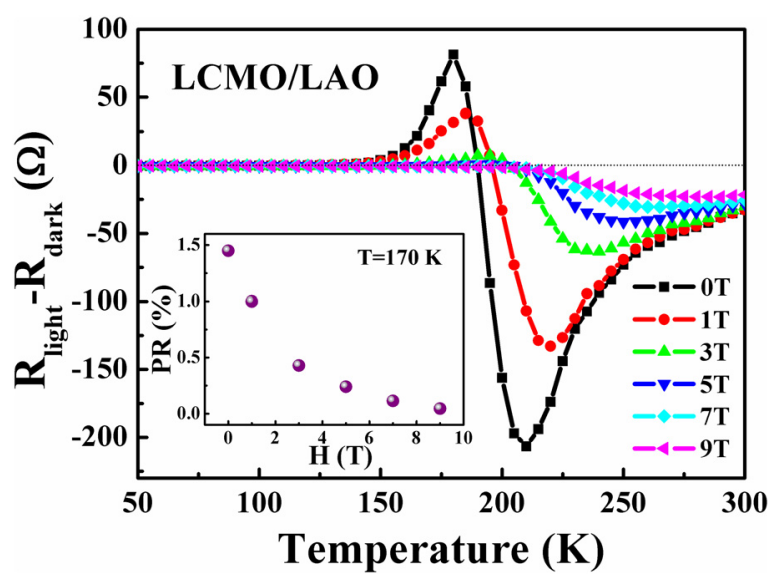

FIG. 4. (Color online) The temperature dependence of $R_{\text {light }}-R_{\text {dark }}$ for LCMO films grown on LAO substrate under various fields of $0,1,3,5,7$, and $9 \mathrm{~T}$, respectively. The inset shows the field dependence of the PR measured in $170 \mathrm{~K}$. stripe-like domains, whereas the domains in the thicker samples (over $135 \mathrm{~nm}$ ) will break up to bubble-like entities. The effects of magnetic fields to the different domain features are thought to be distinct; thus, the degree of suppression of PR in manganite films with various thicknesses may make a difference. In our work, LCMO films with thickness of $\sim 150 \mathrm{~nm}$ may only cover one situation here. Further studies on the different domain characteristics to the suppression of PR are under way.

The PR observed in our work is much smaller than what was reported by Moshnyaga et al. due to the weak light density of $\sim 5 \mathrm{~mW} / \mathrm{cm}^{2}$. In the Ref. 12, an $\mathrm{Al}_{2} \mathrm{O}_{3}$ :Ti pulsed laser with power of $1 \mathrm{~W}$ and focused spot of about $50 \mu \mathrm{m}$ was used as light source. The power density of them is much higher than our He-Ne laser. Therefore, a giant negative photoconductivity in $\mathrm{La}_{0.7} \mathrm{Ca}_{0.3} \mathrm{MnO}_{3}$ thin film can be observed. Recently, Smolyaninova et al. ${ }^{13}$ reported that large photoinduced conductivity reduction in manganite films using a continuous wave argon ion laser with power density of $\sim 31.8$ $\mathrm{mW} / \mathrm{cm}^{2}$. The relative change of film resistance is about $3 \%$ at $110 \mathrm{~K}$, which is comparable with our work. The little differences may be caused by the defects or carrier density of different manganite films. Furthermore, one might suspect that the transition was driven by the laser heating. However, we did not observe any PR or the decrease of magnetization when the LCMO film was irradiated by a $1.55 \mu \mathrm{m}$ semiconductor laser with the power density of $15 \mathrm{~mW} / \mathrm{cm}^{2}$. Since the photon energy $(\sim 0.8 \mathrm{eV})$ is smaller than the band gap of LCMO $(\sim 1 \mathrm{eV})$, and no photoexcited carriers can be generated. The results demonstrate that the PR behavior in our case is induced by its intrinsic property of manganites instead of heating effect.

In conclusion, we performed systematic studies on the effects of magnetic field on the photoinduced demagnetization effect in LCMO films. We observed that the photoinduced demagnetization effect is weakened by the magnetic field in LCMO thin films. In the FMM phase, the decrease of magnetization was observed under light illumination, indicating that the photo-excitation of spin polarized electrons from the localized $t_{2 \mathrm{~g}}$ state to the travelling $e_{\mathrm{g}}$ state can decrease the magnetization of LCMO thin films. It has been also found that the PR can be decreased by external magnetic field. This behavior can be explained by the following two effects: (i) the reorientation of the FMM domains and spin direction of photoexcited carriers in LCMO films and (ii) the release and recombination of electrons trapped around oxygen vacancies under magnetic field. Our results indicate the results of magnetic field can weaken the effects of injection of photoexcited $e_{\mathrm{g}}$ carriers in manganites and make the resistance return to its original values of no light irradiation. It provides us some insight into the nature of physical mechanism of manganites under irradiation of light. The interplay of the external fields is a good demonstration of the strong coupling between spins and charges in such colossal magnetoresistance materials. From the view of potential application, this may be useful in developing practical magnetically tunable photosensors or magneto-optical recording devices.

This work has been supported by the National Natural Science Foundation of China and the National Basic 
Research Program of China, and also granted by a grant of the Research Grant Council of Hong Kong (Project No. HKU702409P), the URC of the University of Hong Kong.

${ }^{1}$ C. Zener, Phys. Rev. 81, 440 (1951).

${ }^{2}$ A. P. Ramirez, P. Schiffer, S.-W. Cheong, C. H. Chen, W. Bao, T. T. M. Palstra, P. L. Gammel, D. J. Bishop, and B. Zegarski, Phys. Rev. Lett. 76, 3188 (1996).

${ }^{3}$ P. Schiffer, A. Ramirez, W. Bao, and S.-W. Cheong, Phys. Rev. Lett. 75, 3336 (1995).

${ }^{4}$ Z. G. Sheng, J. Gao, and Y. P. Sun, Phys. Rev. B 79, 014433 (2009).

${ }^{5}$ K. H. Ahn, T. Lookman, and A. R. Bishop, Nature 428, 401 (2004); C. Thiele, K. Dörr, S. Fähler, L. Schultz, D. C. Meyer, A. A. Levin, and P. Paufler, Appl. Phys. Lett. 87, 262502 (2005); R. K. Zheng, Y. Wang, H. L. W. Chan, C. L. Choy, and H. S. Luo, Appl. Phys. Lett. 90, 152904 (2007). ${ }^{6}$ E. J. Guo, J. Gao, and H. B. Lu, Appl. Phys. Lett. 98, 081903 (2011).

${ }^{7}$ K. Miyano, T. Tanaka, Y. Tomioka, and Y. Tokura, Phys. Rev. Lett. 78, 4257 (1997).

${ }^{8}$ R. Cauro, A. Gilabert, J. P. Coutour, R. Lyonnet, M.-G. Medici, J.-C. Grenet, C. Leighton, and I. K. Shuller, Phys. Rev. B 63, 174423 (2001).
${ }^{9}$ Y. G. Zhao, J. J. Li, R. Shreekala, H. D. Drew, C. L. Chen, W. L. Cao, C. H. Lee, M. Rajeswari, S. B. Ogale, R. Ramesh, G. Baskaran, and T. Venkatesan, Phys. Rev. Lett. 81, 1310 (1998).

${ }^{10}$ K. Matsuda, A. Machida, Y. Moritomo, and A. Nakamura, Phys. Rev. B 58, R4203 (1998).

${ }^{11}$ Z. G. Sheng, Y. P. Sun, J. M. Dai, X. B. Zhu, and W. H. Song, Appl. Phys. Lett. 89, 082503 (2006).

${ }^{12}$ V. Moshnyaga, A. Giske, K. Samwer, E. Mishina, T. Tamura, S. Nakabayashi, A. Belenchuk, O. Shapoval, and L. Kulyuk, J. Appl. Phys. 95, 7360 (2004).

${ }^{13}$ V. N. Smolyaninova, G. Yong, R. M. Kolagani, A. Biswas, H. K. Ermer, K. Wang, and A. Piazza, Appl. Phys. Lett. 99, 222507 (2011).

${ }^{14}$ Q. L. Zhou, K. Zhao, K. J. Jin, D. Y. Guan, H. B. Lu, Z. H. Chen, G. Z. Yang, A. Li, and H. K. Wong, Appl. Phys. Lett. 87, 172510 (2005).

${ }^{15}$ Q. L. Zhou, K. J. Jin, K. Zhao, D. Y. Guan, H. B. Lu, Z. H. Chen, and G. Z. Yang, Phys. Rev. B 72, 224439 (2005).

${ }^{16}$ E. J. Guo, J. Gao, and H. B. Lu, Appl. Phys. Lett. 98, 081903 (2011).

${ }^{17}$ Y. Wu, Y. Suzuki, U. Rüdiger, J. Yu, A. D. Kent, T. K. Nath, and C. B. Eom, Appl. Phys. Lett. 75, 2295 (1999).

${ }^{18}$ S. R. Bakaul, W. Lin, and T. Wu, Appl. Phys. Lett. 99, 042503 (2011).

${ }^{19}$ S. R. Bakaul, W. Lin, and T. Wu, Appl. Phys. Lett. 100, 012403 (2012). 